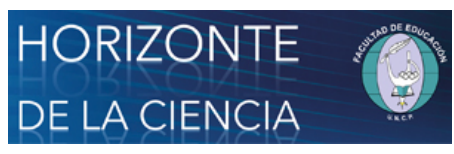

Horizonte de la Ciencia

ISSN: 2413-936X

horizontedelaciencia@uncp.edu.pe

Universidad Nacional del Centro del Perú

Perú

\title{
Importancia de la familia: Un análisis de la realidad a partir de datos estadísticos
}

Diaz Dumont, Jorge Rafael; Ledesma Cuadros, Mildred Jénica; Diaz Tito, Luis Pablo; Tito Cárdenas, Julia Victoria

Importancia de la familia: Un análisis de la realidad a partir de datos estadísticos

Horizonte de la Ciencia, vol. 10, núm. 18, 2020

Universidad Nacional del Centro del Perú, Perú

DOI: https://doi.org/10.26490/uncp.horizonteciencia.2020.18.407

Los autores otorgan el permiso a compartir y usar su trabajo manteniendo la autoría del mismo. Atribución no comercial (CC BY-NC) 4.0 


\section{Importancia de la familia: Un análisis de la realidad a partir de datos estadísticos}

Importance of the family: An analysis of reality based on statistical data

Importância da família: uma análise da realidade a partir de dados estatísticos

Ayllup allikaynin:kaynincichpa likapayninkuna chalkachi kaakunawan

\section{IROPEROTATSIRI ABAKAYETACHARI : KOÑATAKOTACHENERI IROPEROTATSIRI OINIJANTERORI OBAKOYETAKOTACHARI MAROJEITENI}

Kara okomeiti omatsigengasonori: Anetsëkotagekero kara pinagetiri sangenarentsipage Ogokoitobakema

Jorge Rafael Diaz Dumont Datos de los autores

Universidad César Vallejo, Perú

jorge.diazdu@ciplima.org.pe

(iD) http://orcid.org/0000-0003-0921-338X

Mildred Jénica Ledesma Cuadros

Universidad César Vallejo, Perú

iD http://orcid.org/0000-0001-6366-8778

\section{Luis Pablo Diaz Tito}

Universidad Privada San Juan Bautista, Perú

iD http://orcid.org/0000-0001-7602-7638

Julia Victoria Tito Cárdenas

Ministerio de Educación del Perú, Perú

(iD) http://orcid.org/0000-0001-5344-2345
DOI: https://doi.org/10.26490/ uncp.horizonteciencia.2020.18.407

Recepción: 06 Mayo 2019

Aprobación: 08 Junio 2019

Recepción: 06 Mayo 2019

Aprobación: 08 Junio 2019

\section{Resumen:}

La presente investigación tiene como objetivo general explicar la importancia que tiene familia como grupo social en una realidad estadística del Perú. La investigación es de enfoque cualitativo - cuantitativo, se realizó un rastreo de información documental que aborda la importancia de la familia como grupo social; luego se aborda la realidad estadística en el Perú. Los resultados indican que dentro de los grupos sociales la familia es considerada la más importante, en este grupo social se estrechan una serie de lazos que se deben comprender según el tiempo en que fueron dados. En el caso del Perú, las estadísticas reflejan que existe una crisis en la familia, así se da que la situación conyugal informal de convivencia viene incrementándose. Por otro lado, la situación conyugal de casados decrece. Asimismo, la situación conyugal de separados se incrementa; siendo tendencias que debilitan la cohesión familiar.

\section{Notas DE AUTOR}

Datos Jorge Díaz Dumont. Doctor Ingeniero Industrial, Universidad Nacional Federico Villarreal. Licenciado en Educación, Facultad de Teología de los Pontificia y Civil de Lima. Magister en Docencia y Gestión. Doctor en Educación. PhD en Public Administration y Bachelor of Sciencewith a Major autores in Statistics en USA. Está calificado como Investigador en Ciencia y Tecnología del SINACYT - CONCYTEC - Registro N ${ }^{\circ} 15697$ (REGINA). Docente Universidad César Vallejo, Universidad Nacional Autónoma de Tayacaja "Daniel Hernández Morillo" - Perú.

Mildred Jénica Ledesma Cuadros. Doctora en Administración de la Educación por la Universidad César Vallejo. Magister en Docencia y Gestión Educativa. Licenciada en Educación Primaria por la Universidad Nacional Federico Villarreal.

Julia Victoria Tito Cárdenas. Doctora en Educación por la Universidad César Vallejo. Maestra en Docencia Universitaria por la Universidad Nacional Federico Villarreal. Magister en Educación con mención en Docencia y Gestión Educativa. Licenciada en Educación.

jorge.diazdu@ciplima.org.pe 
Palabras Clave: matrimonio, divorcio, concubinato, Ishkaytakuy, lakinakuy, kuskakay, abakantsi, okabakantsi, itsipamishitashitaro, agobagantsi, jokobagantsi, jinatsarintagantsi.

\section{ABSTRACT:}

The present investigation has like general aim to explain the importance that has family like social group in a statistical reality of Peru. The research is of a qualitative-quantitative approach, a documentary information tracking was carried out that addresses the importance of the family as a social group; and then the statistical reality in Peru is addressed. The results indicate that within the social groups the family is considered the most important, in this social group a series of bonds are narrowed that must be understood according to the time they were given. In the case of Peru, the statistics show that there is a crisis in the family, thus the informal conjugal situation of coexistence is increasing. Likewise, the conjugal situation of separated ones increases; being trends that weaken family cohesion.

KEYWORDS: marriage, divorce, concubinage.

\section{Resumo:}

Esta pesquisa tem como objetivo geral explicar a importância da família como grupo social na realidade estatística do Peru. A pesquisa é de abordagem qualitativa - quantitativa, foi realizado um rastreamento de informações documentais que aborda a importância da família como grupo social; depois se aborda a realidade estatística no Peru. Os resultados indicam que, dentro dos grupos sociais, a família é considerada a mais importante; nesse grupo social se estreitam uma série de laços que devem ser compreendidos de acordo com o tempo que foram dados. No caso do Peru as estatísticas mostram que há uma crise na família, do mesmo modo, que a situação conjugal informal de convivência tem aumentado. Por outro lado, a situação de pessoas casadas diminui. Além disso, a situação conjugal de separados se incrementa; sendo tendências que enfraquecem a coesão familiar.

Palavras-Chave: casamento, divórcio, concubinato.

\section{INTRODUCCIÓN}

La familia es el grupo social, en el que se estrechan una serie de lazos que han sido característicos en el transcurrir del tiempo y la historia; sin embargo, estas tienen características especiales, dependiendo de la época y contexto cultural. Dentro del grupo familiar, hay funciones y tareas que se desempeñan, entre las cuales se pueden mencionar: la comunicación y los objetivos comunes; siendo que estos juegan un papel trascendental para que la familia llegue a ser exitosa.

En este orden de ideas se puede afirmar que lo que se entiende por familia (constructo del término), considerando una línea de tiempo, ha cambiado. Si analizamos la prehistoria, se tenía propiamente un jefe de “clan”, más no de grupo familiar; al respecto Acevedo (2011) refirió que:

Primitivamente los seres humanos vivieron en promiscuidad sexual, el heterismo. Tales relaciones excluyen toda posibilidad de establecer con certeza la paternidad, por lo que la filiación sólo podía contarse por línea femenina. A consecuencia de este hecho, se llegó al dominio femenino absoluto, es decir, la "ginecocracia”. El paso a la monogamia en la que la mujer pertenece a un solo hombre, encerraba la transgresión de una antiquísima ley religiosa, es decir, el derecho inmemorial que los demás hombres tenían sobre aquella mujer. (p. 152)

Como vemos propiamente en la prehistoria, la familia como tal no existía, menos aún entendida, como lo es actualmente; sin embargo cabe resaltar que conforme el hombre ejercía cierto dominio sobre la mujer (amparado en la religión) se fue gestando la idea de familia. En el otro extremo, tenemos que actualmente la familia es entendida como al grupo social en el que pueden existir o no lazos biológicos, y en el que el ser humano aprende una serie de comportamientos, adquiere valores; y en donde los lazos afectivos son determinantes para su cohesión.

En la actualidad, la familia, es reconocida por el Estado; siendo que esta, establece ciertas formas de conformación y organización de las mismas (matrimonio, divorcio, concubinato, etc.). Por tanto, en el caso del Perú, las estadísticas existentes sobre estos aspectos, pueden darnos un alcance de su realidad. 
La presente investigación platea como problema fundamental la siguiente pregunta general ¿Cuál es la importancia que tiene familia como grupo social en una realidad estadística del Perú?; en este contexto, se plantea el objetivo general explicar la importancia que tiene familia como grupo social en una realidad estadística del Perú.

\section{Antecedentes del Problema}

Nuestra sociedad sufre una serie de problemas como son: discriminación, feminicidio, racismo, contaminación, guerras, drogadicción, pandillaje, entre otras; el grupo social familiar cobra importancia. La familia, su forma de constitución, su comunicación, sus valores, su éxito en general, puede contribuir como parte de la solución, en cada uno de estos problemas. Sin embargo, se sabe que en la actualidad según Solis y Aguiar (2017):

La familia ha perdido muchas de sus funciones. La irrupción de nuevas agencias de socialización es parte de la solución a los problemas que encierra el capitalismo en la modernidad. Se ha dado un aumento exponencial de guarderías y casas hogar; se ha disminuido la edad para la educación formal; se dan cada vez más contratos entre niñeras y empleadores. Las nuevas generaciones quedan desplazadas de los proyectos de vida comunitarios familiares y de la autoridad de los jefes de familia (p. 2).

Esta problemática actual existente a nivel mundial, perjudica la realización a cabalidad de las funciones que le corresponden asumir a la familia, sin embargo, ello no imposibilita totalmente el ejercicio de las funciones familiares para con la sociedad. Es por ello, que las familias deben preocuparse por tener momentos de calidad juntos, además es preciso preparar a los hijos para enfrentarse a los problemas sociales existentes, buscando alternativas de solución que les permitan afrontar y superar las dificultades que se les presenten en su contexto real.

Asimismo, Solis y Aguiar (2017) refirieron que se debe formar en el niño tanto el aspecto cognitivo, cultural, social y emocional para que en un mediano plazo funcione como una persona competente, productiva, cuidadosa y responsable con la sociedad. Siguiendo la línea, Rodríguez (2010) afirmó: "La familia entonces deberá proporcionar el primer y más importante contexto social, emocional, interpersonal, económico y cultural para el desarrollo humano y, como resultado, las relaciones tendrán una profunda influencia sobre el bienestar de los niños" (p.439). Es en el seno de la familia donde las personas adquieren competencias, hábitos, costumbres, comportamientos, etc.; que les permitirán ser personas de bien y afrontar cada uno de los problemas que se les presenten en su realidad social y tengan las herramientas para poder superarlas y/o solucionarlas en beneficio propio y de la sociedad. Es la familia el espacio donde se trabaja la prevención de conductas inadecuadas, control de emociones, resolución de problemas, autoestima, valores; los cuales servirán para formar una sociedad justa.

Igualmente Ortiz, Torres y Padilla (2005) sostuvieron:

La familia tiene la tarea de desarrollar determinadas funciones, tales como la biológica, la económica, la educativa en la formación de sus integrantes, contribuyendo a la formación de valores, a la socialización de sus miembros, a la educación, a la reproducción y a la satisfacción de necesidades económicas entre otros. Asimismo, tiene la tarea de preparar a los individuos para enfrentar cambios que son producidos tanto desde el exterior como desde el interior y que pueden conllevar a modificaciones estructurales y funcionales. (párr. 5)

La familia tiene un rol primordial en el desarrollo económico, educativo, laboral y social. Esta reflexión lleva a comprender que en la dinámica familiar se debe evaluar en forma constante los problemas existentes dentro de la sociedad con la finalidad de planificar estrategias y mecanismos que permitan enfrentar los desajustes sociales, mitigar sus efectos y preparar a los integrantes para formar una sociedad equitativa, íntegra donde se busque el bien común. 


\section{CONCEPTO DE FAMILIA}

El concepto de familia desde el punto de vista histórico ha ido evolucionando tanto en su estructura, tipos como en sus roles. Desde una concepción tradicional se considera a la familia como el grupo de individuos los cuales se encuentran entrelazados por vínculos de amor y sangre, en la cual la unión en matrimonio de los padres es duradera y los hijos son educados en el seno del hogar. Sin embargo, esta concepción no es la única, por su parte, Martínez (2015) afirmó que la familia es considerada como la primera forma de organización social y su existir se corrobora en los testimonios históricos de cada uno de los grupos sociales de las diferentes épocas. Asimismo, Martínez (2015) refirió que desde el ámbito filosófico la familia es considerada como una categoría histórica, la manera en la cual se encuentra organizada está condicionada por el imperante régimen económico y/o social y por las relaciones sociales existentes. Desde la concepción sociológica según Oliva y Villa (2013) se considera a la familia como un grupo cuya característica principal es la realización de la relación sexual definida y permanente con el fin de promover la procreación y la educación de la prole. Así mismo, Oliva y Villa (2013) afirmaron que la familia en la actualidad es "considerada como un núcleo natural, económico y/o jurídico de la sociedad" (p.14)

Entonces, el concepto de familia no tiene una única connotación, esta ha ido evolucionando de manera significativa, pero, "en principio sigue siendo la célula de la sociedad humana" (Acevedo, 2011, p.149). La familia es la célula principal de la sociedad, es el grupo de personas que conforman un hogar, donde no necesariamente todos se encuentran unidos por lazos consanguíneos pero si de afecto y amor. En el seno de la familia se cultivan los primeros valores éticos que servirán como cimientos para el desarrollo de la sociedad.

\section{LA FAMILIA COMO GRUPO SOCIAL, EN LA HISTORIA}

Las familias desde sus orígenes han ido evolucionando de un estadio a otro, cambiando su dinámica y la concepción. A través de la historia se observa diferentes formas de organizaciones familiares cada una con sus características peculiares. Los primeros modelos de familia en la prehistoria se dieron a través de la sociedad matriarcal y patriarcal, luego se desarrolló la familia en la sociedad Babilónica, en el imperio Egipcio, en la sociedad Hebrea, en la Grecia clásica hasta llegar a la familia actual. Respecto a la sociedad matriarcal, Acevedo (2011) sostuvo que en la sociedad matriarcal se vivió en promiscuidad sexual, debido a ello la filiación se contaba únicamente por la línea femenina y es así que la mujer llegó a tener máximo dominio (ginecocracia), dentro de esta sociedad matriarcal se observaron algunos tipos de familias. Al respecto, Valladares (2008) mencionó la siguiente clasificación: familia consanguínea, la cual constituyó la primera forma de relación entre personas de diferente sexo, esta unión no estaba basada en el matrimonio, sino en una necesidad netamente sexual donde se desconocen los vínculos parentales; familia punalúa, este tipo de familia se desarrolló en la época del salvajismo, las relaciones íntimas se encontraban limitadas únicamente a los integrantes de los clanes o tribus; familia sindiásmica, en este tipo de familia hubo la presencia de una pareja, pero es permitido para los hombres tener relaciones sexuales con otras integrantes de su grupo, los hijos aún continúan perteneciendo a la madre. En la sociedad patriarcal, según Acevedo (2011) debido a que la sociedad matriarcal presentaba algunas debilidades en el desarrollo de la civilización se dio paso a la sociedad patriarcal la cual se encontraba enfocado en los valores masculinos y en el desarrollo del derecho civil. En esta sociedad se dio paso a la familia monogámica, en la cual tuvo predominio la imagen masculina, convirtiéndose en el dueño de la familia. Siguiendo la línea de Valladares (2008) en la familia monogámica, se dio el matrimonio, se originó la propiedad privada y se reconoció la paternidad de manera indiscutible con la finalidad de heredar los bienes a sus descendientes.

Referente a las familias en la antigüedad, Acevedo (2011) sostuvo que la familia en la sociedad babilónica se encontraba amparada por el matrimonio, es decir era monogámica, pero el esposo tenía la autorización de tener esposas secundarias en caso la esposa principal no pudiera tener hijos, el rol principal del matrimonio era 
la procreación y la conservación de la familia. En la sociedad egipcia se conservó el matrimonio monogámico, el cual se celebra a través de ceremonias religiosas, solamente el faraón podía tener varias esposas. En la sociedad hebrea, el matrimonio jurídicamente tenía carácter civil, en esta sociedad el esposo podía tener varias esposas legítimas y también concubinas. En la Grecia clásica, la célula principal de la sociedad estaba conformada por la familia, cuya base era el matrimonio o en algunos casos el concubinato, es decir era monogámica, careciendo la mujer de derechos ciudadanos y políticos, cuando era hija se encontraba sometida a su padre, cuando llegaba a ser esposa a su marido y cuando era viuda quedaba sometida a los hijos.

Tal es así, que la familia existió desde tiempos prehistóricos y se conservan hasta nuestros días. En la actualidad, por lo general, se habla de la familia ideal conformada por padres e hijos cuando en la realidad existen diversos modelos de familias las cuales poseen sus propias características de acuerdo a su conformación, formas de vida y de interrelación entre sus miembros.

Oliva y Villa (2013) refirieron que:

Debido a la confluencia de intrínsecos aspectos a su naturaleza como lo son el aspecto histórico, político, socio-cultural y por el desarrollo psico-afectivo de sus miembros, cada familia es única y diferente, no sólo por las relaciones, roles y el número de personas que la forman, sino también por las actividades y trabajos que realizan o la manera en que se organizan y proyectan. (p.12)

En las últimas décadas el concepto de familia ha ido evolucionando aún más, tal es así que la mirada por aquellas familias nucleares conformadas únicamente por padres e hijos se ha transformado y se ha dado paso a las otras formas de familias las cuales se pueden encontrar conformadas por lazos sanguíneos, matrimonio, adopciones, etc. Al respecto, Del Pico (2011) sostuvo que la familia, sin importar la connotación que se le asigne realiza diversas funciones en la sociedad, su valoración pública determina la tutela jurídica la cual se expresa en el Derecho de Familia. La familia, sin importar la definición, su naturaleza osu clasificación, goza de derechos; asimismo, se encuentra amparada por acuerdos internacionales y documentos oficiales como La Declaración Universal de los Derechos Humanos (1948) el cual en el artículo 16 considera a la familia como un elemento natural y fundamental de la sociedad y afirma su derecho a ser protegida tanto por la sociedad como por el Estado. Por su parte, Congreso de la República (1993) en la Constitución Política del Perú refirióen el capítulo II, artículo 4 la protección a la familia. Como se observa no se hacen distinciones por algún tipo de familia en particular sino que la consideran tal cual está constituida, es decir en forma integral.

Existen diferentes formas de clasificar a las familias, teniendo en cuenta la forma como están constituidas. Según Benítez (2008) las familias pueden clasificarse en: familia nuclear, la cual se encuentra conformada por el padre, la madre y los hijos; familia extensa, este tipo de familia se encuentra conformada por los integrantes de más de dos generaciones las cuales se encuentran unidas por vínculos sanguíneos, incluye padres, hijos, abuelos, tíos, sobrinos, primos, etc.; familia monoparental, este tipo de familia se encuentra conformada únicamente por uno de los padres y los hijos, esta se da debido al divorcio o al fallecimiento de uno de los padres; familia de madre soltera, en este tipo de familia es la madre la única que asume desde un inicio con la responsabilidad de la crianza del hijo; familias de padres separados, en este tipo de familia los padres rompen su relación de pareja pero no se desligan de los hijos, es decir no rehúyen a su paternidad y maternidad.

Esta clasificación corresponde al presente siglo debido a que existen diferentes formas y tipos de familia las cuales tienen sus propios valores, costumbres y forma de vida y ello no debería cambiar en absoluto la valoración social que se le asigna ya que cumple con su función social.

\section{IMPORTANCIA DE LA FAMILIA COMO GRUPO SOCIAL}

La familia como grupo social posee gran importancia puesto que contribuye en la formación integral de cada uno de sus miembros en especial de los hijos. Al respecto, Besanilla y Miranda (2013) sostuvieron que la persona desde que nace se encuentra en el seno familiar y es allí donde vive, se desarrolla y adquiere habilidades 
las cuales le serán necesarias en los diferentes ámbitos de su vida. Por otra parte, Pérez (1999) afirmó que en la familia se halla protección e intimidad, pero para que estas se pongan en manifiesto es necesario que los integrantes aprendan a expresar sus sentimientos con libertad. La característica mencionada no es innata, por el contrario es adquirida, por ello, es necesario ser practicada hasta lograr alcanzarla.

De la influencia que ejerzan los padres y de la cohesión de sus integrantes dependerá la vida familiar ya que el hogar es el primer espacio donde se aprende los valores, costumbres, normas, conductas, etc. es por ello importante que en la familia se cultive el amor, el respeto mutuo, la consideración, etc., aceptando y respetando la cultura de su grupo y planificando proyectos de vida familiar. Sin embargo, no siempre la realidad es la expuesta ya que también actualmente existen familias quebradas por causa del divorcio, el cual es considerado según Tamez y Ribeiro (2016) como un fenómeno social y familiar el cual consiste en la separación del esposo y esposa convirtiéndose el hogar en un espacio monoparental. En la familia monoparental uno de los integrantes, por lo general la madre, es quien asume la mayor responsabilidad del cuidado y educación de los hijos, siendo dicho espacio donde iniciarán los hijos su vida social e irán aprendiendo las primeras formas de convivencia e interrelaciones. Otra forma de familia que también se debe tomar en cuenta son aquellas donde los padres formalizaron el compromiso de conformar una nueva familia sin llegar al matrimonio, es decir a través del concubinato, al respecto Espinoza (2015) refirió que el concubinato en estos tiempos modernos se ha convertido en una alternativa para regular la vida en pareja. Estamos frente a una "unión de hecho" la cual le otorga a la familia los mismos beneficios legales que un matrimonio formalmente constituido. Este tipo de familia también tiene gran importancia en la sociedad ya que en el seno del hogar se formarán los futuros ciudadanos impregnados de los valores y costumbres que la familia le brinde.

Como se observa en la sociedad actual existe una multiplicidad de modelos de familia cada uno con sus características peculiares pero si con una misma misión que es la de formar ciudadanos capaces de aportar a la sociedad de la cual forman parte y en ello es que radica la principal importancia de la familia en el siglo actual.

\section{FUNCIONES Y TAREAS DEL GRUPO FAMILIAR}

Uno de los elementos primordiales que caracterizan a las familias son las funciones que realizan los integrantes, para así lograr una dinámica adecuada y el bienestar familiar. Besanilla y Miranda (2013) mencionaron que la familia tiene como responsabilidad principal brindar educación y formación a sus integrantes con la finalidad que desarrollen su potencial y puedan insertarse dentro del sistema social de manera adecuada y productiva. La forma en la que la familia cumpla sus funciones dependerá del contexto sociocultural y del entorno en el cual se desenvuelven. Otra de las funciones, tal vez la más importante, es la función afectiva puesto que esta permite el desarrollo de la inteligencia emocional, la mejora de la autoestima y brindan protección a la salud integral de cada uno sus miembros. Pi Osoria y Cobian (2009) sostuvieron que la función afectiva de la familia, es un valor primordial y se centra en la relación entre dicha función y la vida sana de los integrantes. Es por ello, la importancia de cultivar una vida saludable en familia a fin de que cada uno de sus integrantes se desarrolle en un ambiente agradable, de amor, concordiay unidad.

\section{ReALiDAD ESTADÍSTiCA}

Habiéndose explicado la importancia que tiene la familia como grupo social, además de definirla y clasificarla; es importante presentar la realidad estadística (respecto a la familia) que existe en el Perú, ello permitirá tener un matiz particular a nuestra sociedad. 
Como se observa en la tabla 1 y figura 1, la población en el Perú, desde los años $1940(703,111)$ al año 2017 (31'237,385), ha presentado una importante tendencia creciente, lo que representa un incremento del $344.8 \%$.

TABLA 1

Población en el Perú

\begin{tabular}{ll}
\hline Año & Total \\
\hline 1940 & 7023111 \\
1961 & 10420357 \\
1972 & 14121564 \\
1981 & 17762231 \\
1993 & 22639443 \\
2007 & 28220764 \\
2017 & 31237385 \\
\hline
\end{tabular}

Fuente: INEI - Censos Nacionales de Población y Vivienda: 1940, 1961, 1972, 1981, 1993, 2007 y 2017.

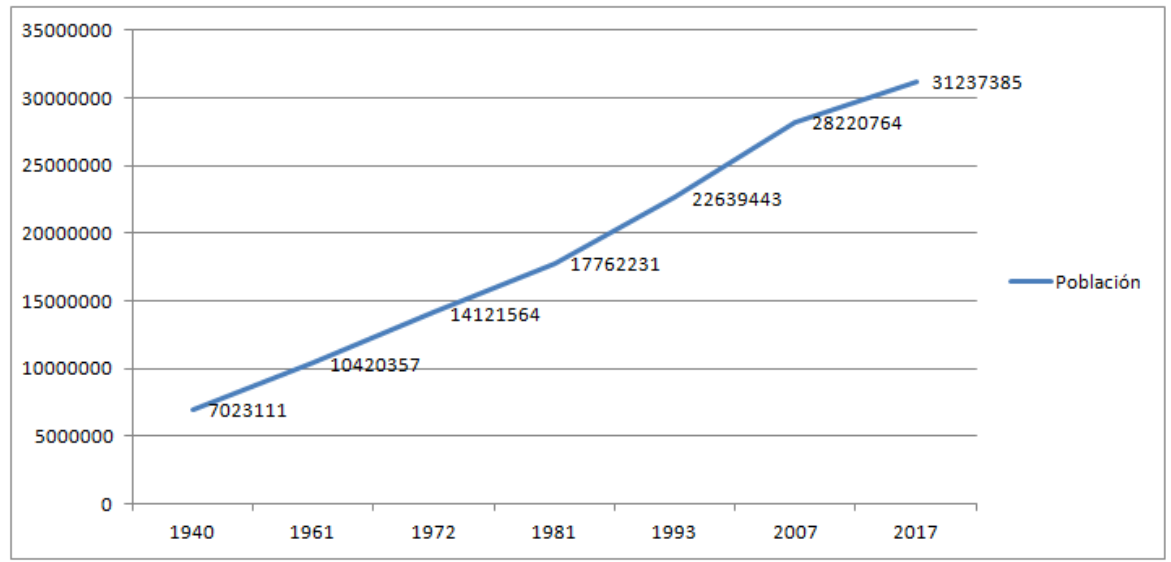

FIGURA 1

Gráfico lineal de la población en el Perú

Elaborado por los autores, 2019

Igualmente en la tabla 2 y figura 2, se observa que la población de mujeres durante los años 2007 (50.3\%) y 2017 (50.8\%), ha sido superior al de los hombres; habiéndose variado ligeramente en un $0.5 \%$ en cuanto al total de la población.

TABLA 2

Población Censada según sexo

\begin{tabular}{lllll}
\hline & 2007 & $\%$ & 2017 & $\%$ \\
\hline Hombres & 13622640 & $49.7 \%$ & 14450757 & $49.2 \%$ \\
Mujeres & 13789517 & $50.3 \%$ & 14931127 & $50.8 \%$ \\
TOTAL & 27412157 & $100.0 \%$ & 29381884 & $100.0 \%$ \\
\hline
\end{tabular}

Fuente: INEI - Censos Nacionales de Población y Vivienda 2007 y 2017 


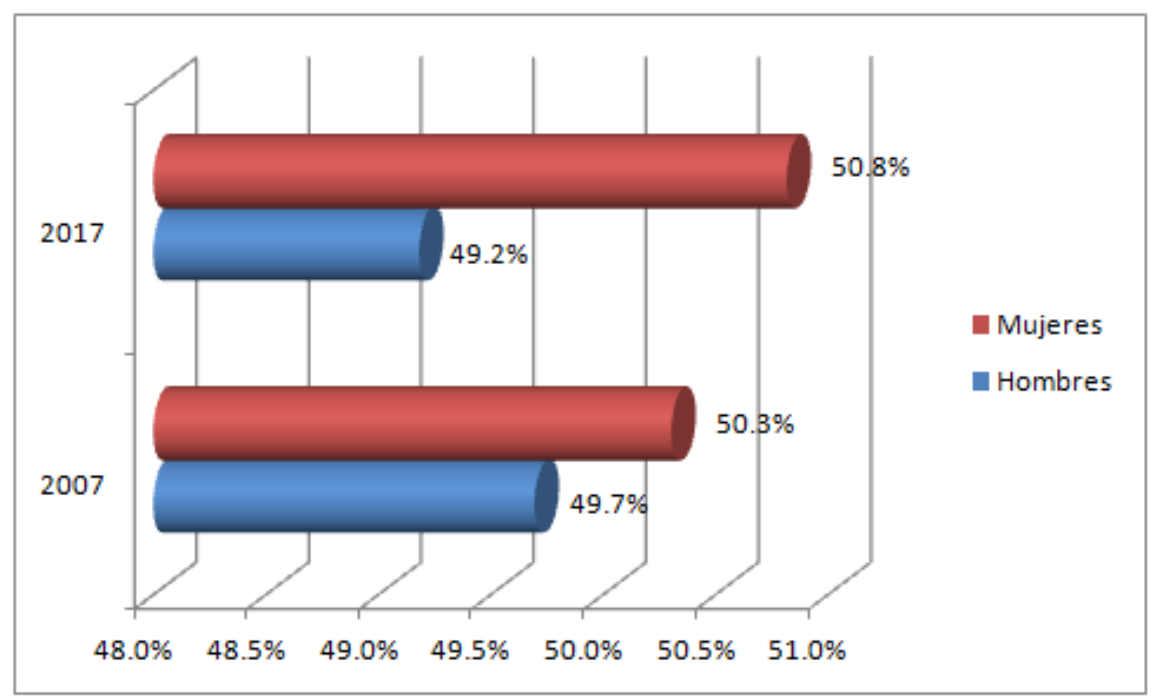

FIGURA 2

Gráfico de barras agrupadas de la población según sexo

La situación conyugal o estado civil presentada en la tabla 3 y figura 3, presenta una realidad respecto a la situación conyugal de convivencia, esta se incrementó desde el censo de 2007 de 24.6\% (5 124925) al censo del 2017 en 26.7\% (6 195 795); ello en contraposición de la situación conyugal de casado, esta decreció desde el censo de 2007 de $28.6 \%$ (5 962 864) al censo del 2017 en $25.7 \%$ (5 959 966). Por otro lado, la realidad respecto a la situación conyugal de separados, esta se incrementó desde el censo de 2007 de 3.4\% (714 242) al censo del 2017 en $4.2 \%$ (968 413).

\section{TABLA 3}

Perú: Población censada de 12 y más años, según estado civil o conyugal, 2007- 2017

\begin{tabular}{lllll}
\hline Estado civil o & \multicolumn{2}{l}{ Censo 2007 } & \multicolumn{2}{c}{ Censo 2017 } \\
\cline { 2 - 5 } Conyugal & Absoluto $\%$ & \multicolumn{3}{l}{ Absoluto $\%$} \\
\hline Total & 20850 & 100,0 & 23196 & 100,0 \\
& 502 & \multicolumn{3}{l}{391} \\
Conviviente & 5124 & 24,6 & 6195 & 26,7 \\
& 925 & & 795 & \\
Separado/a & 714242 & 3,4 & 968413 & 4,2 \\
Casado/a & 5962 & 28,6 & 5959 & 25,7 \\
& 864 & & 966 & \\
Viudo/a & 809707 & 3,9 & 940437 & 4,1 \\
Divorciado/a & 114093 & 0,5 & 209707 & 0,9 \\
Soltero/a & 8124 & 39,0 & 8922 & 38,5 \\
& 671 & & 073 & \\
\hline
\end{tabular}

Fuente: INEI - Censos Nacionales de Población y Vivienda, 2007 y 2017 


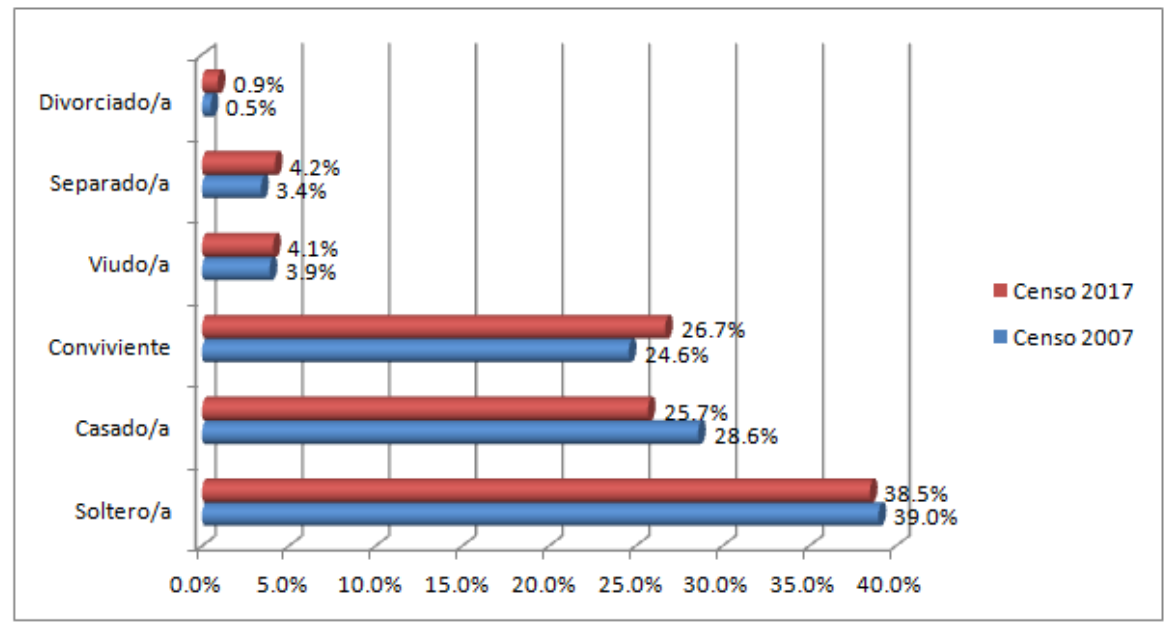

IGURA 3

Gráfico de barras agrupadas estado civil o conyugal

Elaborado por los autores, 2019

En la tabla 4, presenta la situación del estado civil o conyugal por grupo de edad según el censo Nacional de Población y Vivienda 2017, refleja algunas situaciones particulares.

TABLA 4

Perú: Situación del estado civil o conyugal por grupo de edad al 2017

\begin{tabular}{lllllllll}
\hline $\begin{array}{l}\text { Grupo } \\
\text { de }\end{array}$ & Total & \multicolumn{7}{l}{ Estado civil o conyugal } \\
\cline { 3 - 8 } edad & & Total & Conviviente & Separado/a & Casado/a & Viudo/a & Divorciado/a & Soltero/a \\
\hline Total & $23,196,391$ & 100.0 & 26.7 & 4.2 & 25.7 & 4.1 & 0.9 & 38.5 \\
$12-14$ & $1,568,558$ & 100.0 & 0.3 & 0.003 & 0.001 & - & - & 99.7 \\
$15-19$ & $2,422,478$ & 100.0 & 6.9 & 0.4 & 0.3 & 0.013 & 0.009 & 92.3 \\
$20-24$ & $2,508,736$ & 100.0 & 27.7 & 2.0 & 3.5 & 0.1 & 0.1 & 66.7 \\
$25-29$ & $2,386,320$ & 100.0 & 42.1 & 3.3 & 11.2 & 0.2 & 0.2 & 43.1 \\
$30-34$ & $2,257,102$ & 100.0 & 46.4 & 4.6 & 20.8 & 0.3 & 0.4 & 27.5 \\
$35-39$ & $2,122,675$ & 100.0 & 43.5 & 5.6 & 28.9 & 0.6 & 0.7 & 20.7 \\
$40-44$ & $1,952,661$ & 100.0 & 38.4 & 6.4 & 35.4 & 1.1 & 1.2 & 17.6 \\
$45-49$ & $1,707,717$ & 100.0 & 32.5 & 7.0 & 41.0 & 1.8 & 1.6 & 16.1 \\
50 y & $6,270,144$ & 100.0 & 16.7 & 5.8 & 49.8 & 13.8 & 2.1 & 11.9 \\
más & & & & & & & & \\
\hline
\end{tabular}

Fuente: INEI - Censos Nacionales de Población y Vivienda. 2017 


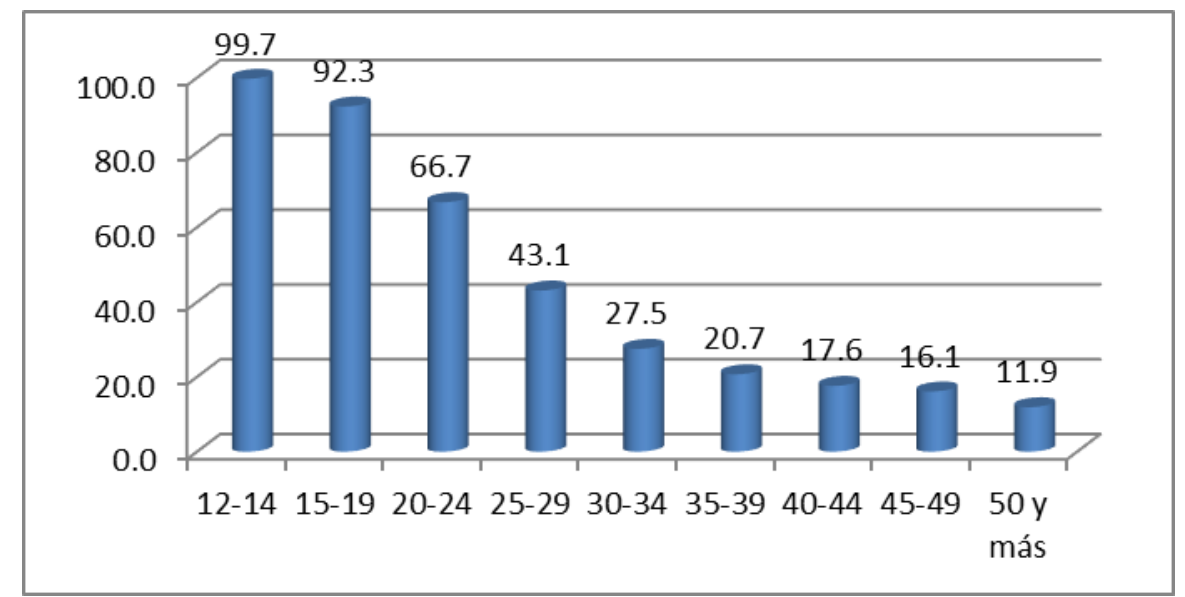

\section{FIGURA 4}

Soltero/a por Grupo de edad censo del 2017

En la figura 4, se refleja una situación decreciente en cuanto al estado civil de soltero (a), a medida que se avanza en la edad, decrece la soltería, como es lógico, puesto que la familia se consolida de 99.7\% (12-14 años de edad) a $11.9 \%$ (50 a más años).

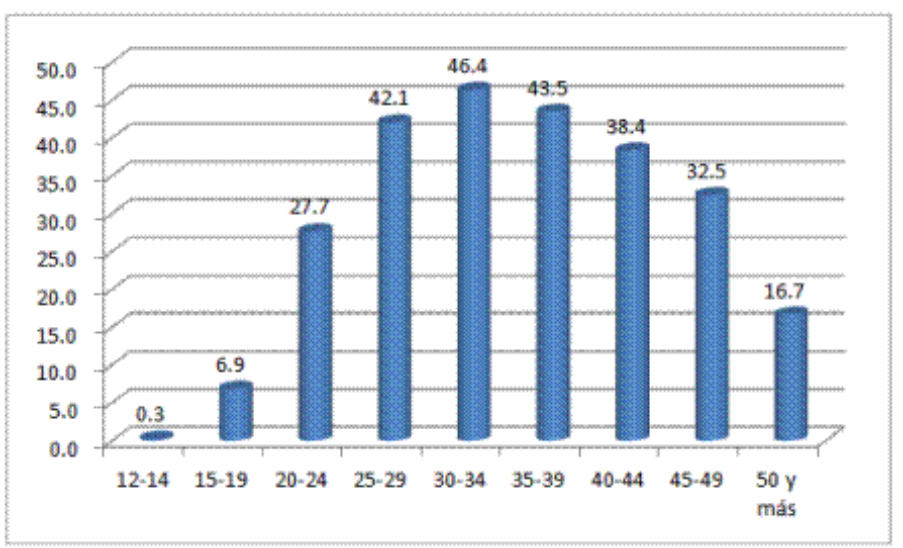

FIGURA 5

Conviviente por grupo de edad censo del 2017

Sin embargo en la figura 5 , en contraposición, se observa que la situación de convivencia y no formalidad tiene una marcada situación entre los grupos de edades de 20 hasta los 34 años de edad, para ir descendiendo o formalizándose desde los 35 a más. 


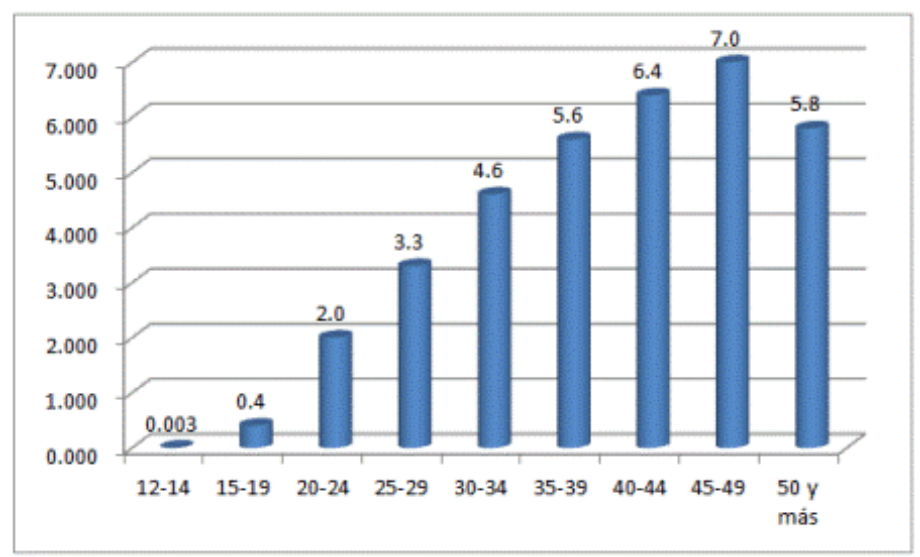

FIGURA 6

Separado por grupo de edad censo del 2017

Igualmente, en la figura la situación conyugal de separado (a), hasta los 49 años existe una tendencia a la ruptura de la familia, en contraposición cuando llega la edad de los 50 años donde la tendencia es decreciente.

\section{Discusión}

En cuanto a las conclusiones tenemos que:

Dentro de los grupos sociales "la familia" es uno de los más importantes, en este grupo social se estrechan una serie de lazos, que hay que entenderlos y comprenderlos según el tiempo que fueron dados. Asimismo, estos tienen características especiales, dependiendo de la época y contexto cultural.

Los primeros modelos de familia en la prehistoria se dieron a través de la sociedad matriarcal y patriarcal.

En las sociedades actuales la familia es reconocida por el Estado, el cual establece las diversas formas de conformación y organización formal.

La familia como grupo social posee gran importancia ya que contribuye en la formación integral de cada uno de sus miembros en especial de los hijos; en este sentido, muchos de los problemas que afronta la sociedad tienen su explicación en la crisis que atraviesa la familia.

En el caso del Perú las estadísticas reflejan que existe una crisis en la familia, así se da que la situación conyugal informal de convivencia viene incrementándose. Por otro lado, la situación conyugal de casados decrece. Así mismo, la situación conyugal de separados se incrementa; siendo tendencias que debilitan la cohesión familiar.

A partir del análisis proponemos las siguientes recomendaciones:

A. Es necesario promover la importancia de la familia como tal ya que su fortalecimiento contribuirá a solucionar diversos problemas que afectan a la sociedad; para ello, es necesario tratar el tema desde la escuela y a través de campañas institucionales por parte del Estado.

B. Las cifras estadísticas reflejan una realidad preocupante respecto a la familia, por ello, es necesario difundirlas y explicarlas en diversos foros y paneles.

\section{REFERENCIAS BIBLIOGRÁFICAS}

Acevedo, L. (2011). El concepto de familia hoy. Francisanum LIII (156), 149 - 170. Recuperado en 26 de febrero de 2019, de http://www.scielo.org.co/pdf/frcn/v53n156/v53n156a06.pdf 
Asamblea General de Naciones Unidas (1948) Declaración Universal de los Derechos Humanos. Recuperado de https://www.minjus.gob.pe/wp-content/uploads/2014/03/DECLARACION-UNIVERSALDE-DERECHOS-HUMANOS.pdf

Benítez M. (2008). La estructura familiar en La familia cubana en la segunda mitad del siglo xx. La Habana: Editorial Ciencias Sociales.

Bezanilla, J. y Miranda, A. (2013). La familia como grupo social: una re-conceptualización. Alternativas en Psicología, 17(29), 58-73. Recuperado en 26 de febrero de 2019, de http://pepsic.bvsalud.org/scielo.php? script $=$ sci_arttext\&pid=S1405-339X2013000200005\&lng=pt\&tlng=es.

Congreso de la República (1993). Constitución Política del Perú. Recuperado de https://www.oas.org/juridico/ spanish/per_res17.pdf

Del Picó, J. (2011). Evolución y actualidad de la concepción de familia: una apreciación de la incidencia positiva de las tendencias dominantes a partir de la reforma del derecho matrimonial chileno. Lus et Praxis, 17(1), 31-56. https://dx.doi.org/10.4067/S0718-00122011000100003

Espinoza, Á. (2015). La juridificación de las uniones de hecho y la propuesta valórica contenida en el proyecto legislativo de Acuerdo de Vida en Pareja en Chile. Lus et Praxis, 21(1), 101-135. https://dx.doi.org/10.4067/ S0718-00122015000100004

INEI (2019). Perfil Socio demográfico del Perú 2017. Recuperado de: https://www.inei.gob.pe/media/ MenuRecursivo/publicaciones_digitales/Est/Lib1539/index.html

Martínez, H. (2015). La familia: una visión interdisciplinaria. Revista Médica Electrónica, 37(5), 523-534. Recuperado en 25 de febrero de 2019, de http://scielo.sld.cu/scielo.php? script=sci_arttext\&pid=S1684-18242015000500011\&lng=es\&tlng=es.

Oliva, E. y Villa V. (2013). Hacia un concepto interdisciplinario de la familia en la globalización. Justicia Juris, 10 (1), 11-20. Recuperado en 25 de febrero de 2019, de http://www.scielo.org.co/pdf/jusju/v10n1/v10n1a02.pdf

Ortiz, M. Torres, M. y Padilla, E. (2005). Diseño de intervención en funcionamiento familiar. Psicologia para América Latina, (3) Recuperado en 27 de febrero de 2019, de http://pepsic.bvsalud.org/scielo.php? script $=$ sci_arttext\&pid $=$ S1870-350X2005000100005\&lng=pt\&tlng=es.

Pérez, C. (1999). La familia en su etapa de formación. Revista Cubana de Medicina General Integral, 15(3), 237-240. Recuperado en 26 de febrero de 2019, de http://scielo.sld.cu/scielo.php? script $=$ sci_arttext\&pid=S0864-21251999000300003\&lng=es\&tlng=es.

Pi Osoria A. y Cobián, A. (2009) Componentes de la función afectiva familiar: una nueva visión de sus dimensiones e interrelaciones. Medisan ;13(6). Recuperado en 26 de febrero de 2019, de http://bvs.sld.cu/revistas/san/ vol13_6_09/san16609.htm

Rodríguez, M. (2010). Factores personales y familiares asociados a los problemas de comportamiento en niños. Estudios de Psicología, Campinas 27(4) 437-447. Recuperado de http://www.scielo.br/pdf/estpsi/ v27n4/02.pdf

Solís, F. y Aguiar, R. (2017). Análisis del papel del involucramiento de la familia en la escuela secundaria y su repercusión en el rendimiento académico. Sinéctica, (49) Recuperado en 27 de febrero de 2019, de http:// www.scielo.org.mx/scielo.php?script=sci_arttext\&pid=S1665-109X2017000200013\&lng=es\&tlng=es.

Tamez, B. y Ribeiro, M. (2016). El divorcio, indicador de transformación social y familiar con impacto diferencial entre los sexos: estudio realizado en Nuevo León. Papeles de población, 22(90), 229-263. https:// dx.doi.org/10.22185/24487147.2016.90.040

Valladares, A. (2008). La familia. Una mirada desde la Psicología. Revista Médica Electrónica, 6(1), 3-13. Recuperado en 25 de febrero de 2019, de http://soda.ustadistancia.edu.co/enlinea/ mariaespinosa_bienestarpsicologicoyfamiliar/4c615f66616d696c69612e5f556e615f6d69726164615f64657364655f6c615f

Los autores otorgan el permiso a compartir y usar su trabajo manteniendo la autoría del mismo. CC BY-NC 